\title{
Homéopathie Classique - Pratique et recherche 2012
}

\author{
Suite de notre série d'articles sur les quatre sociétés de médecine complémentaire \\ de I'UNION: c'est à présent au tour du concept de traitement homéopathique d'être \\ présenté.
}

\author{
Martin Frei-Erba, \\ Klaus von Ammon a, \\ Barbara Kramer ${ }^{b}$ \\ Clemens Dietrich ${ }^{c}$ \\ a Kollegiale Instanz für \\ Komplementärmedizin \\ KIKOM, Université de Berne \\ b Spécialiste en médecine \\ générale $\mathrm{FMH}$, AFC en \\ homéopathie SSMH \\ c Président de la Société Suisse \\ des Médecins Homéopathes \\ $\mathrm{SSMH}$
}

* Les références se trouveront sous www.bullmed.ch

$\rightarrow$ Numéro actuel ou

$\rightarrow$ Archives $\rightarrow 2012 \rightarrow 40$
¿Correspondance: Dr Clemens Dietrich Rigistrasse 5 CH-5610 Wohlen Tél. 0566229277
La Société Suisse des Médecins Homéopathes (SSMH/SVHA) fut fondée en 1856 et représente ainsi une des sociétés médicales les plus traditionnelles de la Suisse [1]*. De nos jours, elle englobe aussi des médecins dentistes, des vétérinaires et des pharmaciens/-nes. Depuis l'introduction du certificat de capacité en 1999 reconnue par la FMH, la société a enregistré une croissance importante au niveau de ses membres; actuellement, elle compte 280 membres médecins dont 253 possédent le certificat de compétence, ce qui représente la majorité des médecins homéopathes actifs en Suisse.

\section{Homéopathie Classique}

Le terme d'homéopathie est souvent employé pour désigner tout traitement finissant par la prescription d'un remède homéopathique. Mais, les sels de Schüssler, les fleurs de Bach et les remèdes homéopathiques complexes,c'est-à-dire des remèdes conte- semble à une anamnèse bio-psycho-sociale et sera complétée par des informations spécifiques à l'homéopathie afin de saisir la totalité des symptômes. S'ensuit l'examen physique du patient, si nécessaire complété par le laboratoire et/ou l'imagerie. Grâce à toutes ces informations, le médecin en possession d'un certificat de compétence en homéopathie pose toujours deux diagnostics: un diagnostic conventionnel et un diagnostic homéopathique. Ensuite, un plan thérapeutique individuel est établi en fonction de la pertinence des problèmes.

L'ensemble des symptômes homéopathiques recueillis par rapport à un remède (Materia medica) a été constitué par des expérimentations de la substance dynamisée auprès de personnes en bonne santé $[2,3]$, par les données toxicologiques et finalement par l'expérience clinique provenant de patients traités avec succès. En analogie avec l'image de la maladie (la totalité des symptômes), cet ensemble

\section{«Le médecin en possession d'un certificat de compétence en homéo- pathie pose toujours deux diagnostics: un diagnostic conventionnel et un diagnostic homéopathique.»}

nant plusieurs substances dynamisées homéopathiquement n'ont pas de point commun avec l'Homéopathie Classique, mis à part le fait qu'il s'agit d'administrer des remèdes dynamisés. De même, la prescription de remèdes homéopathiques en fonction du diagnostic clinique ne répond pas aux règles de l'Homéopathie Classique. Ces fausses idées conduisent à des malentendus et des résultats de traitement et de recherche insatisfaisants.

L'Homéopathie Classique [2] est une méthode de soins pour le traitement des maladies qui a fait ses preuves à travers le monde au cours des deux siècles précédents. Le but interventionnel en Homéopathie Classique n'est pas défini ni par des maladies spécifiques ni par des symptômes individuels. Afin de trouver le remède individualisé d'un patient, l'anamnèse complète est indispensable; cette dernière res- d'informations fournit l'image d'un remède homéopathique. La procédure de l'expérimentation homéopathique moderne correspond à la phase I d'une étude médicamenteuse en médecine conventionelle.

Le choix du remède se fait selon le principe de similitude (similia similibus curentur:que le semblable se guérisse par le semblable). Ce qui veut dire qu'une maladie peut être guérie par le remède qui ressemble dans la totalité des symptômes le mieux aux symptômes produits par le patient. Par conséquence, lorsqu'un patient présente plusieurs diagnostics conventionnels, en Homéopathie Classique un seul remède lui est prescrit par consultation.

Le principe de la similitude n'est pas spécifique à l'homéopathie mais a été décrit auparavant par Hippocrate et Paracelse. Même la médecine conventionnelle en fait usage, par exemple dans le cas du 


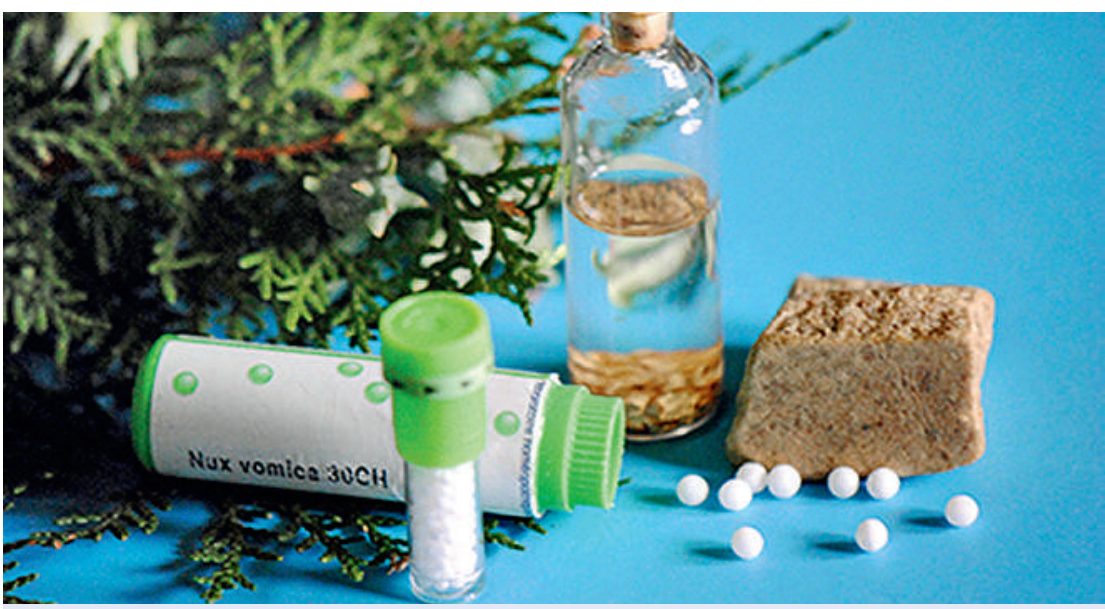

Les médicaments homéopathiques sont fabriqués à partir de substances végétales, animales ou minérales. traitement du syndrome ADHS chez les enfants et adolescents (syndrome de déficit d'attention et d'hyperactivité) en leur prescrivant le Méthylphenidate.

Les remèdes homéopathiques sont produits à partir de substances végétales, animales et minérales, parfois aussi à partir d'agents pathogènes (nosodes); ils sont en général absorbés par voie orale sous forme de granules ou de gouttes. Une nomenclature spécifique définit les médicaments dynamisés: On parle de dynamisations décimales ou «dilutions D» lorsque la substance de base est diluée progressivement en proportion de 1:10; si les étapes de dilution

\section{«L'efficacité de l'intervention homéopathique a été démontrée dans plusieurs contextes cliniques.»}

se font dans une proportion de 1:100, on parle de dynamisations centésimales ou «dilutions $\mathrm{C}$ » [2].

\section{L'homéopathie dans la pratique médicale}

Grâce à son emploi varié et son indépendance d'une infrastructure, grâce à son absence de toxicité et sa bonne tolérance, grâce aussi au fait qu'elle peut être combinée avec d'autres interventions thérapeutiques, l'Homéopathie Classique est une forme de thérapie très adaptée à la médecine de premier recours. Tout médecin ayant une autorisation de pratique cantonale peut pratiquer l'homéopathie sans aucune limitation sans devoir faire preuve d'une formation. Afin de garantir la qualité de la formation homéopathique des médecins, l'Association des Médecins suisses FMH a mis en place en 1999 un certificat de capacité pour l'homéopathie [4]. Les cours de formation ont lieu à Berne, Lausanne, Lucerne et Zurich [5]. Les médecins ainsi formés peuvent, grâce à leur compétence médicale et à leur complément de formation en homéopathie, décider de cas en cas et dans l'entente avec le patient, si un traitement conventionnel seul, un traitement homéopathique d'accompagnement ou un traitement homéopathique seul est possible et indiqué. Depuis le 1.1.2012, les prestations homéopathiques peuvent être facturées dans l'assurance de base par les médecins porteurs d'un certificat de capacité.

\section{Homéopathie Classique et Critères EAE}

Le programme d'évaluation de la médecine complémentaire PEK 1999-2005 de l'OFSP et des associations de médecine complémentaire suisses constitue une œuvre de pionnier dans le domaine Health Technology Assessment HTA en Suisse [6]. Son exécution constituait pour toutes les personnes concernées un grand défi avec divers méandres et confusions méthodologiques et politiques. Néanmoins, grâce à la masse d'information recueillie, plusieurs publications ont vu le jour qui confirment la place de la médecine complémentaire et en particulier de l'Homéopathie Classique comme une méthode adéquate et économique dans l'assurance de base [7]. La dernière de ces études compare dans une analyse transversale les coûts de 562 médecins de premier recours, avec ou sans certificat de capacité, entre 20022003; il démontre des coûts plus bas de $15,4 \%$ chez les porteurs du certificat de capacité en homéopathie [8]. Ces résultats sont confirmés par une étude récente provenant des Pays-Bas [9].

Dans le cadre de la demande de réadmission de l'homéopathie dans l'assurance de base faite par la SSMH en 2010, une recherche de littérature concernant l'efficacité de l'homéopathie a été effectuée en novembre 2009 en complément des données PEK. L'efficacité de l'Homéopathie Classique, déjà démontrée en 2006 par le HTA Homéopathie [10], se confirme à travers des études plus récentes: Dans 7 des 11 études randomisées contrôlées, l'homéopathie individualisée montre une meilleure efficacité [11-21]. L'efficacité de l'intervention homéopathique a été démontrée dans plusieurs contextes cliniques dont le syndrome ADHS chez les enfants et adolescents [13]. Vu depuis la perspective de la médecine intégrative, les deux travaux de Frass sont remarquables: L'homéopathie comme complément thérapeutique dans un service de médecine intensive avec des patients atteints de sepsis et de COPD $[12,22]$.

En conclusion, on peut dire que les traitements homéopathiques individualisés se caractérisent par un large spectre d'indication au sein de la médecine de premier recours et sont au moins aussi efficaces que la médecine conventionnelle; dans les maladies chroniques à long terme, grâce au setting orienté vers le patient, grâce aussi à l'absence de toxicité et au faible coût, leur bénéfice est notable. 